\title{
The Anatomical Foundations of Regional Anesthesia and Acute Pain Medicine
}

\author{
Andre P. Boezaart (editor). Bentham Science Publishers, 2016. ISBN 978-1-68108-192-2
}

\author{
Rakesh V. Sondekoppam, MD
}

Received: 19 July 2016/ Accepted: 8 August 2016/Published online: 15 August 2016

(C) Canadian Anesthesiologists' Society 2016

A strong foundation in anatomy is paramount for the safety and success of regional anesthesia. In his 1924 book, Regional Anesthesia: Its Technique and Clinical Application, Dr. Gaston Labat noted that, "A thorough knowledge of descriptive and topographic anatomy with regards to nerve distribution is a condition which anyone desirous of attempting to study regional anesthesia should fulfill," aptly emphasizing the importance of anatomical understanding for those practicing regional anesthesia. The popularity of ultrasonographic guidance for regional anesthesia, which affords a better appreciation of the anatomy of interest, the needle-nerve interaction, and drug deposition, makes these anatomical foundations all the more important. There are currently only a few textbooks dedicated specifically to regional anesthesia anatomy, and the present The Anatomical Foundations of Regional Anesthesia and Acute Pain Medicine by Andre P. Boezaart is an excellent, welcome addition to this small collection. This textbook attempts to bridge the gap in the compendium of regional anesthesia literature by providing a comprehensive review of the micro-anatomy and macroanatomy relevant to procedures in regional anesthesia and pain medicine. The editor and the authors of the book are recognized experts in the specialty and have helped advance the specialty of regional anesthesia.

This 384-page book is available primarily as an e-book, with an option of print-on-demand from the publisher. The book contains a total of 23 chapters that are well organized into logical sections of the upper limb, lower limb, trunk,

R. V. Sondekoppam, MD ( $\square)$

Department of Anesthesia and Pain Medicine, University of Alberta, Edmonton, AB, Canada

e-mail: vijayash@ualberta.ca head and neck, and neuraxial blocks. All of the chapters have been authored or co-authored by the editor, which is reflected in the consistent flow of the text. The block regions have been presented in a uniform format, first with a detailed description of the macro-anatomy followed by the micro-anatomy. The corresponding sonographic and functional anatomy follow the gross anatomical description for each region. Each chapter begins with an introductory outline. The in-depth anatomical description for each region reflects the exhaustive experience, abundant academic expertise, and comprehensive knowledge of the author(s). The list of references in each chapter are up to date.

Chapters 1-9 mainly focus on upper limb anatomy, with Chapters 2 and 4 addressing the micro-anatomy of the nervous system in general. These two chapters are a must read for any budding regional anesthesiologist as the basic concepts of neuronal architecture and its relation to drug spread is well explained. Chapter 2 provides an in-depth explanation of drug diffusion across the membranes as well as a description of cerebrospinal fluid flow and its importance to regional anesthetic blocks. Accompanying images of the central and intrafascicular spread of the dye and functional micro-anatomy makes it easy to understand the text.

Chapters 10-17 cover lower limb anatomy. These chapters begin with a description of the macroarchitecture and the microstructure of each nerve along with the osteotomal and dermatomal anatomy. This is followed by the innervation of the adjoining bones and relevant joints. The chapters covering the femoral and sciatic nerves offer anesthesiologists the knowledge and rationale for combining various nerve blocks for lower limb surgery. 
Chapters 18, 19 and 20 cover the anatomy relevant to blocks on the trunk, and Chapter 21 covers neuraxial anatomy. Anatomy relevant to the sympathetic nervous system and the nerves important to head and neck blocks are described in Chapters 22 and 23. The macroscopic and microscopic anatomy are covered along with relevant fluoroscopic/sonographic anatomy in Chapters 18-23, unlike separate chapters for each section as appeared in the chapters on the upper and lower limbs. These chapters are succinct and convey anatomical information important for performing and troubleshooting regional blocks.

A key highlight of the book is the comprehensive collection of images and videos that also serve as a practical atlas of anatomy relevant to regional anesthesia. There are more than 200 images describing the gross anatomy, the ultrastructure and course of nerves, and the sonographic anatomy, along with images of the dermatomal, osteotomal, and neurotomal distributions, all of which contribute to easy reading of the accompanying text. Indeed, the well-edited videos significantly enhance the learning experience.

Despite the book's strengths, some areas for improvement exist. For example, there is considerable overlap of content between the macro-anatomy and functional anatomy in the sections on the upper and lower limbs. The sequence of macro-anatomy followed by micro-anatomy, sonographic anatomy, and functional anatomy is divided into distinct chapters for the upper and lower limbs, but this segregation into different chapters is not consistent after Chapter 17. Highlighting the key points at the beginning of each chapter, perhaps in a future edition, could further improve the textbook.

In summary, the innumerable strengths of this textbook far outweigh its above-noted shortcomings. The Anatomical Foundations of Regional Anesthesia and Acute Pain Medicine could be a valuable reference for all regional anesthesiologists and pain medicine specialists, from novices to the experienced practitioner. Given the textbook's organized structure, in-depth content, and accompanying illustrations, it should easily find a home on a wide array of professional and personal library shelves.

Conflicts of interest None declared.

Editorial Responsibility This submission was handled by $\mathrm{Dr}$. Hilary P. Grocott, Editor-in-Chief, Canadian Journal of Anesthesia. 